\title{
Psicopedagogía crítica para conceptos epistémicos y sociales en investigación universitaria
}

\section{Critical Psychopedagogy for Concepts Epistemic and Social in University Research}

\author{
Psicopedagogia crítica para conceitos epistêmicos e sociais na pesquisa \\ universitária \\ Mario Condori Nina \\ Fundación Universidad del Sur, La Paz - Bolivia \\ mariocondori2015@hotmail.com \\ https://orcid.org/0000-0002-9224-9510
}

\section{Resumen}

El objetivo de este trabajo fue proponer nuevas definiciones de términos epistémicos que son utilizados en el ámbito universitario y particularmente en ciencias socioculturales: gnoseología, epistemología y teoría del conocimiento, por un lado, y por el otro, los términos de explicación y comprensión. Para ello, estos conceptos pasaron por una exposición descriptiva para posteriormente ser tratados psicopedagógica y sociocríticamente, lo cual permitió conocer los pormenores intrínsecos que tienen cada uno ellos; este proceso garantizó una base teórica que permitió y fundamentó la propuesta de nuevas concepciones de los cinco términos en cuestión. Aparte del método de trabajo de campo de investigación, se utilizó el análisis teórico discriminante, el método descriptivo, el inductivo, el análisis-síntesis y de posición, el de la elevación de lo abstracto a lo concreto y lo histórico-lógico; también se recurrió al paradigma sociocrítico, al método propositivo y, particularmente, al método de la filofización de los hechos. Entre los resultados, primero, se logró cumplir con el objetivo de formular nuevas proposiciones conceptuales para los términos epistémicos y sociales ya mencionados; segundo, se detectó que cada uno de los autores revisados tiene una posición conceptual distinta respecto a un solo término universal, lo cual lleva a la confusión; tercero, las tres epistemes de la gnoseología, la 


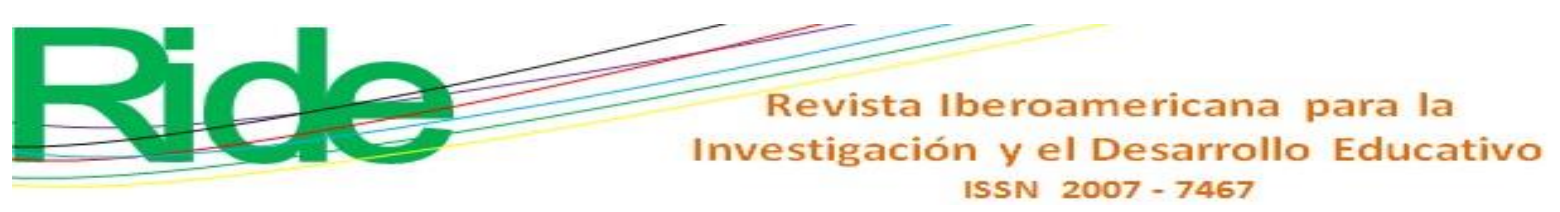

epistemología y la teoría del conocimiento son sinónimas, pero la epistemología se diferencia de las otras dos por ser la única que implica un tratamiento del método; y cuarto, respecto a los términos sociales de explicación y comprensión, ambos pueden contribuir ya sea de manera separada o conjunta a las ciencias físicas, naturales y sociales, pero el último de alguna forma se diferencia del primero porque es más profundo en su actuar en relación con todo tipo de hechos: si bien permite ver cuestiones objetivas, principalmente es útil para temas intersubjetivos y trascendentales de todo tipo de hechos materiales y humanos.

Palabras clave: comprensión, epistemología, explicación, filofización, gnoseología, psicopedagogía, teoría del conocimiento.

\section{Abstract}

The objective of this work was to propose new definitions of epistemic terms that are used in the university environment and particularly in sociocultural sciences: epistemology, epistemology and theory of knowledge, on the one hand, and on the other, the terms of explanation and understanding. To do this, these concepts went through a descriptive exposition to later be treated psychopedagogically and sociocritically, which allowed knowing the intrinsic details that each one has; This process guaranteed a theoretical base that allowed and supported the proposal of new conceptions of the five terms in question. Apart from the research field work method, the discriminant theoretical analysis, the descriptive method, the inductive method, the analysissynthesis and position, the elevation of the abstract to the concrete and the historical-logical were used; The socio-critical paradigm, the propositional method and, particularly, the method of the philosophization of events were also used. Among the results, first, it was possible to meet the objective of formulating new conceptual propositions for the aforementioned epistemic and social terms; second, it was detected that each of the reviewed authors has a different conceptual position regarding a single universal term, which leads to confusion; third, the three epistemes of epistemology, epistemology, and the theory of knowledge are synonymous, but epistemology differs from the other two because it is the only one that implies a treatment of the method; and fourth, regarding the social terms of explanation and understanding, both can contribute either separately or jointly to the physical, natural and social sciences, but the latter in some way differs from the former because it is more profound in its action in relationship with all kinds of facts: 


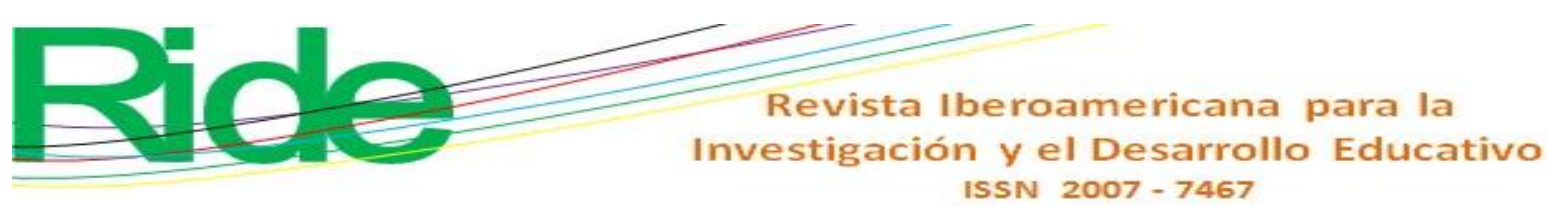

although it allows us to see objective questions, it is mainly useful for intersubjective and transcendental issues of all kinds of material and human facts.

Keywords: understanding, epistemology, explanation, philosophization, gnoseology, psychopedagogy, theory of knowledge.

\section{Resumo}

O objetivo deste trabalho foi propor novas definições de termos epistêmicos utilizados no ambiente universitário e particularmente nas ciências socioculturais: epistemologia, epistemologia e teoria do conhecimento, por um lado, e por outro, os termos de explicação e compreensão. Para isso, esses conceitos passaram por uma exposição descritiva para serem posteriormente tratados psicopedagogicamente e sociocriticamente, o que permitiu conhecer os detalhes intrínsecos que cada um possui; Esse processo garantiu uma base teórica que permitiu e apoiou a proposta de novas concepções dos cinco termos em questão. Além do método de trabalho em campo de pesquisa, foram utilizadas a análise teórica discriminante, o método descritivo, o método indutivo, a síntese-análise e a posição, a elevação do resumo ao concreto e o histórico-lógico; O paradigma sócio-crítico, o método proposicional e, particularmente, o método da filosofização dos eventos também foram utilizados. Entre os resultados, primeiro, foi possível cumprir o objetivo de formular novas proposições conceituais para os termos epistêmicos e sociais acima mencionados; segundo, foi detectado que cada um dos autores revisados tem uma posição conceitual diferente em relação a um único termo universal, o que leva à confusão; terceiro, os três epistemólogos da epistemologia, epistemologia e teoria do conhecimento são sinônimos, mas a epistemologia difere das outras duas porque é a única que implica um tratamento do método; e quarto, com relação aos termos sociais de explicação e compreensão, ambos podem contribuir de forma separada ou conjunta para as ciências físicas, naturais e sociais, mas a última de alguma forma difere da primeira porque é mais profunda em sua ação em relacionamento com todos os tipos de fatos: embora nos permita ver perguntas objetivas, é útil principalmente para questões intersubjetivas e transcendentais de todos os tipos de fatos materiais e humanos.

Palavras-chave: compreensão, epistemologia, explicação, filosofização, gnoseologia, psicopedagogia, teoria do conhecimento. 


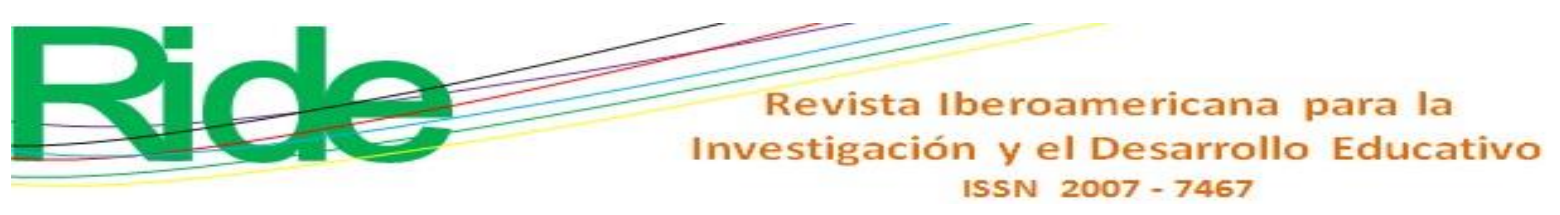

Fecha Recepción: Diciembre 2019

Fecha Aceptación: Mayo 2020

\section{Introducción}

Desde una percepción general de la comunidad universitaria, normalmente el tema de la epistemología se ha entendido como un tema similar a la filosofía, o sea como si ambas fueran difíciles de llegar a entenderse porque cuyos contenidos textuales se asemejan a escritos criptográficos; pero pese a esa percepción, la epistemología es tan relevante para el avance de la ciencia y las interciencias, que por ese motivo no es posible dejar de lado y no hacer tratamiento de conceptos epistemológicos, para ello inicialmente es pertinente recurrir a la psicopedagogía crítica, el cual en este documento permitió analizar y proponer nuevos contenidos de conceptos de los términos epistémicos y sociales: gnoseología, epistemología y teoría del conocimiento, por un lado, y por otro, la explicación y la comprensión. De esa forma en el presente trabajo no solo será actuar como replicador de teorías, puesto que debemos darnos cuenta que somos humanos dotados de diversas potenciales intelectuales que bien pueden servir para repetir mecánicamente las teorías, pero también pueden servir para innovar y crear cosas materiales y hechos no materiales intersubjetivos suscitados en el ámbito de las sociedades. En ese sentido, en el presente documento, se avanza en este tipo de trabajos, o sea, en la innovación y creación propositiva de nuevos conceptos del género epistémico y social.

Por lo expuesto, aquí se entiende que la psicopedagogía puede contribuir al cambio de los temas conceptuales de la epistemología, motivo por el cual, en el siguiente párrafo se expone como ejemplo un adelanto del desarrollo del presente trabajo: el procedimiento de cómo es posible dotarse de un concepto de psicopedagogía que aporte al propósito del presente escrito.

Antes que nada, el título de este artículo exige establecer un significado apropiado de psicopedagogía, para lo cual se procede a separar las palabras que la componen: psicología y pedagogía; luego, de la misma forma, se procede a separar cada uno estos términos aún más con el propósito de demostrar, conocer y establecer el concepto que permitirá orientar el presente artículo. Así, se inicia con la primera palabra, psicología, la cual se puede "quebrar” en dos: psico y logía, el primero significa 'alma' o también 'actividad mental del ser humano', y el segundo significa 'tratado' o 'estudio'. Análogamente, la segunda palabra, pedagogía, también está compuesta y formada por dos términos: paidòs ('niño') y agogè ('conducción'), y en conjunto significan 'niño conducido a la educación escolar', aunque en este caso es el estudiante 


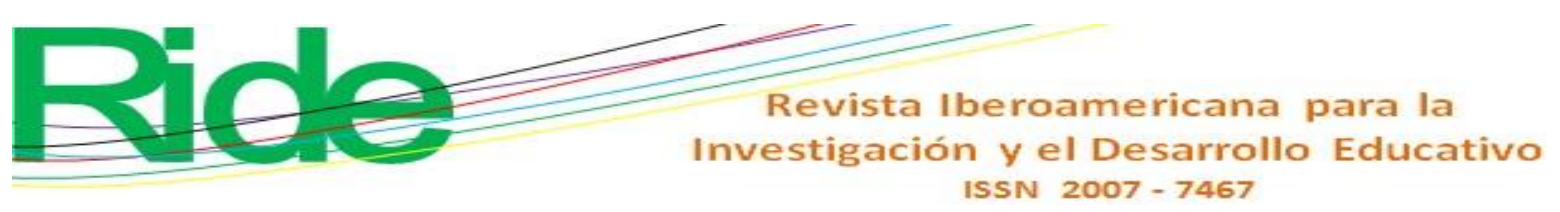

conducido a la educación universitaria. En ese sentido, haciendo la fusión de los significados de ambas palabras, psicología y pedagogía, se obtiene la siguiente oración: "alma o actividad mental humana para la educación y enseñanza". Cabe señalar que se está hablando del ser humano no en sentido de objeto, materia física o anatomía; se está hablando del ser humano desde su componente subjetivo, que impone la forma de relación que deberá tener el cuerpo físico de la persona ante cualquier caso de acción en la sociedad. Por esos motivos, la psicología y la pedagogía, en este artículo, se unen con el propósito de contribuir a demostrar que, con actitud de poseer el alma o espíritu del investigador o escritor científico, dicha persona esté motivada a revisar temas de educación superior como son los conceptos epistémicos y sociales, los cuales son parte de este trabajo. En síntesis, se entiende por psicopedagogía crítica a la ciencia compartida que está presente en la mente, en el alma y en el espíritu del investigador o escritor, cuya esencia y finalidad está destinada a orientar su realización con la actitud mental de investigar, describir, analizar sociocríticamente y proponer nuevos conceptos de términos sociales y de términos epistémicos clásicos que se manejan en la educación superior universitaria.

Por lo expuesto, el presente trabajo de investigación y reflexión filosófica y epistemológica tiene el objetivo de proponer nuevos conceptos de términos epistémicos y sociales que son utilizados en el ámbito universitario y particularmente en ciencias socioculturales. Dicho objetivo se logrará sobre la base del tratamiento de conceptos provenientes de prestigiosos autores de las teorías epistémicas y sociales.

Para finalizar esta parte introductoria, se expone brevemente la estructura de este artículo: a) el método, donde se presentan los seis procedimientos de investigación utilizados para alcanzar el objetivo del presente trabajo; $b$ ) los resultados, cuyo contenido se constituye en la característica de este trabajo, y en donde se aglutinan los temas de dichos resultados, de los cuales posteriormente se realizaron los concernientes análisis sociocríticos para culminar con la elaboración de las respectivas proposiciones, eso como un nuevo procedimiento paradigmático de hacer tratamiento de dichos resultados y las connotaciones holísticas de cada término epistémico y social; por ese motivo, esa forma de proceder es también una novedad incluida de yapa (es decir, de aumento o plus) a la estructura oficial; $c$ ) la discusión y análisis sociocrítico, en este apartado se hace el análisis con base en la episteme del criticismo, y por último, d) la proposición, donde se contribuye con nuevos conceptos a la teoría científica universal, con base en la innovación y la creatividad. 


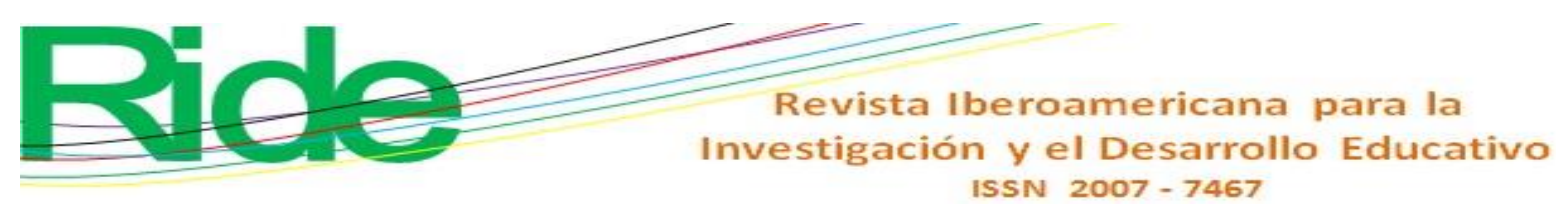

\section{La metódica}

Tratar conceptos epistémicos en sí es una cuestión de carácter teórico, por tanto, para su tratamiento fue pertinente recurrir a métodos de investigación que contribuyeran al proceso de investigación y estudio de los contenidos epistémicos de los términos aquí en cuestión. Por ese motivo, en la presente investigación se estableció tomar en cuenta el siguiente cuerpo metódico:

1) El método de trabajo de investigación de campo, a través del cual se indagó las teorías en diferentes fuentes de información como bibliotecas y centros de fuentes virtuales.

2) El método de análisis de contenido y el análisis teórico discriminante; dicho método, según Delgado y Gutiérrez (1999), destaca en los siguientes términos: Este método pretende describir la singularidad — generalmente semántica - de textos individuales, con frecuencia producidos por autores distintos. Se trata de detectar los rasgos peculiares de cada texto -0 del sujeto al que puede atribuirse-es decir, aquellos que lo identifican por contraposición a los demás. Esos rasgos, o bien son exclusivos del texto -o sujeto- en cuestión, o bien aparecen en este con una frecuencia sensible diferente de la que se detecta en los otros (p. 201).

3) El método descriptivo, que contribuyó a relievar puntual y textualmente las oraciones de los contenidos de los conceptos de los términos epistémicos y sociales.

4) Los métodos teóricos, como la inducción-inducción, el análisis-síntesis y posición, la elevación de lo abstracto a lo concreto y lo histórico-lógico; cada uno de ellos fueron utilizados en el tratamiento teórico de la información obtenida.

5) El método del análisis sociocrítico, que consiste en la actitud psicopedagógica crítica y social que debe poner el investigador en relación con cualquier tema, el cual debe ser contextualizado con la dialéctica histórica de su ámbito social.

6) El método propositivo, que consiste también en recurrir y asumir una actitud psicopedagógica crítica y propositiva a través de la innovación y creación de nuevas teorías. De esa forma, se actuó de manera humana a través de la filofización de los hechos, en este caso, de los hechos teóricos y no solo de manera mecánica, puesto que los temas tratados son de características teóricas, subjetivas y trascendentales. 


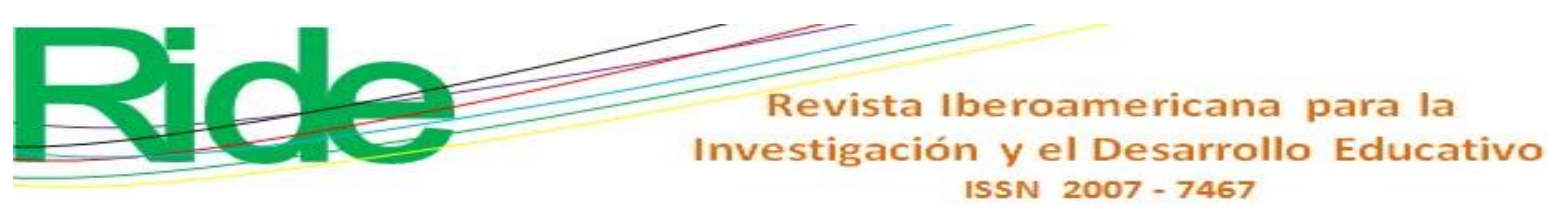

\section{Los resultados, las discusiones sociocríticas y las proposiciones}

Para este apartado se registró abundante información de temas epistémicos generados en diversos tiempos y por diversos autores. La exposición descriptiva del tema fue la base para que, posteriormente, en el siguiente párrafo, se haga la discusión sociocrítica respectiva de cada uno de los contenidos de los conceptos epistémicos y sociales. Por ese motivo, a continuación, se inicia con la descripción de los conceptos de los siguientes términos: gnoseología, epistemología, teoría del conocimiento, comprensión y explicación. Pero antes de proceder vale la pena mencionar algunos puntos respecto a la delimitación, fortaleza y debilidad que presenta este trabajo. En ese sentido, señalar, respecto a la delimitación, que solo se hace tratamiento de cinco conceptos del campo de la episteme y del campo social, pese a que los componentes conceptuales son bastantes en la teoría de ambas áreas; en cuanto a la fortaleza, este trabajo presenta primero aquella de que sé es propositivo, es decir, se propone la innovación y creación de nuevos conceptos, lo que representa un avance en cuanto a los requisitos que exige la presentación de artículos; hay, además, una segunda fortaleza, la cual consiste en que la parte destinada a los resultados se fusiona con el acápite de discusión y, a su vez, esta se fusiona con el tratamiento analítico psicopedagógico sociocrítico, lo cual representa tratar de forma conjunta y holística los temas propuestos. En cuanto a la debilidad del artículo, no existe dicha debilidad, puesto que se logra alcanzar el objetivo de este trabajo.

Una vez expuesto los tres temas señalados, se prosigue con los resultados y el análisis holístico de cada uno de estos.

\section{Del concepto de gnoseología}

Para el análisis y definición conceptual de la palabra gnoseología, primeramente, se recurrió a teorías conceptuales de diversos autores de la filosofía y las ciencias sociales. Entre ellos se tienen a los siguientes autores y sus respectivas conceptualizaciones:

1) Para Álvarez de Zayas (2012), "la gnoseología es la disciplina filosófica que explica el proceso de adquisición del conocimiento" (p. 120).

2) En su versión digital, Encarta (2009) sostiene lo siguiente: Gnoseología (del griego gnosis, 'conocimiento', y logos, 'teoría') es la rama de la filosofía que tiene como pretensión analizar la naturaleza, posibilidad y límites del conocimiento. Asimismo, analiza el problema del origen del conocimiento y de sus formas. La gnoseología estudia los distintos tipos 


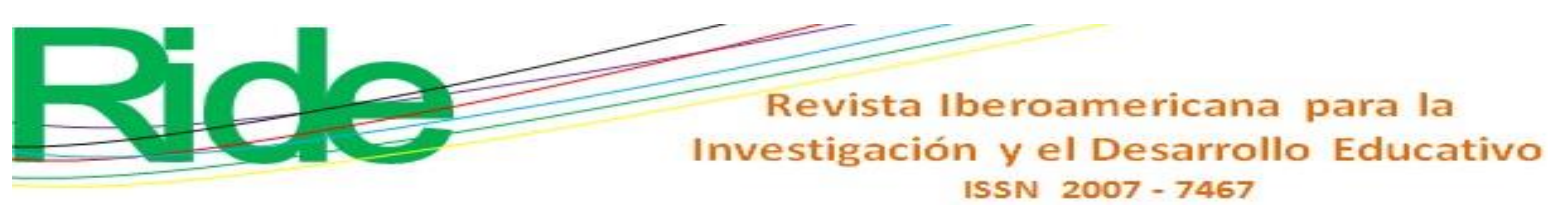

de conocimiento que pueden alcanzarse y el problema de la fundamentación de los mismos. En muchas ocasiones, se identifica con los conceptos teoría del conocimiento o epistemología.

3) Los autores rusos Rosental y Iudin (2007) señalan que la teoría del conocimiento es lo puesto a continuación: Parte importante de la teoría filosófica, versa acerca de la facultad del hombre para entrar en conocimiento de la realidad, acerca de las fuentes de las formas y de los métodos del conocimiento, acerca de la verdad y de los caminos para llegar a conocerla. El término fue introducido por Kant. El punto de partida de la teoría del conocimiento radica en la diversa solución que se da a la cuestión fundamental de la filosofía.

4) Por su parte, Juárez (citado en Sánchez, 1996) sostiene que el término gnosis se debe entender en las siguientes palabras: (Del gr. Gnosis, acción de conocer y logos, tratado). (Fil) Disciplina filosófica que estudia el origen, valor y alcance del conocimiento humano. Históricamente se han utilizado diferentes nombres para expresar este concepto: epistemología, metafísica especial, teoría del conocimiento, criteriología.

Hoy los autores, aunque sin unanimidad total, suelen reservar el de gnoseología para la teoría general del conocimiento, y el de epistemología para el estudio de la naturaleza, valor y alcance de cada una de las ciencias (p. 693).

\section{Discusión y análisis sociocrítico}

Siguiendo estas referencias del término gnoseología, los cuatro autores coinciden en mencionar a la palabra conocimiento como significado de gnoseología. Ampliando sobre dicha palabra, Rosental y Iudin (2007) y Sánchez (1996) señalan que la gnoseología es la teoría del conocimiento e indican, además, que es la teoría general del conocimiento; así, la gnoseología corresponde a la rama de la filosofía que analiza la naturaleza, posibilidad y límites del conocimiento. Aunado a ello, la gnoseología analiza el problema del origen y las formas del conocimiento. Y estudia los distintos tipos de conocimiento y sus respectivos fundamentos.

Desde el enfoque general de la teoría de contenido, en los conceptos de los cuatro autores prevalece o resalta la palabra conocimiento, y no así el término saber, por ese motivo se llega a entender que gnoseología es sinónimo de conocimiento.

Lo que llama la atención de estas cuatro definiciones de gnoseología es que cada uno de los autores dan enfoques diversos sobre un solo término universal. Este hecho causa un problema de cognición y entendimiento, particularmente cuando las interpretaciones son diversas, más cuando el tiempo histórico, el espacio y la época actual son gravitantes para que un concepto sea 


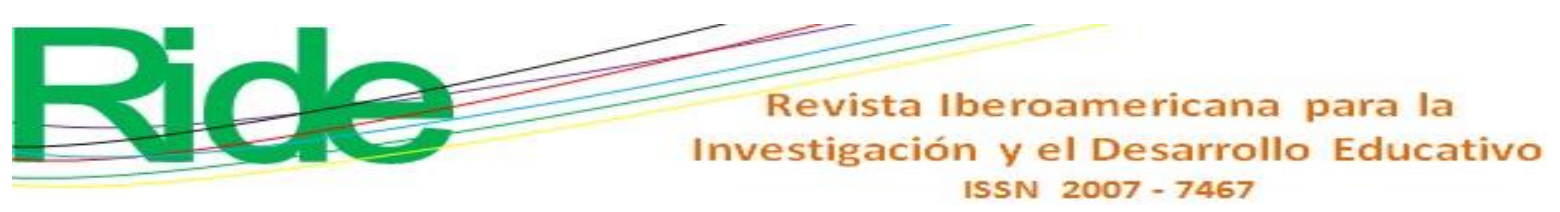

apropiado para la presente época. Por ese motivo, con la finalidad de contribuir a la teoría epistémica, en el siguiente párrafo se presenta la propuesta de definición de gnoseología.

\section{Proposición de definición sobre el concepto gnoseología}

La gnoseología es la teoría filosófica y científica del conocimiento que estudia la naturaleza, la posibilidad, el origen, la esencia, los fundamentos, las clases y los límites del conocimiento humano.

\section{Del concepto de epistemología}

La teoría conceptual de la palabra epistemología es amplia y diversa. Rosental y Iudin (2007, p. 148), Bunge (2007, p. 62), Huanca (2012, p. 30), Conill (citado en Sánchez, 1996, pp. 547-553), Mansilla (2007, p. 48), Tamayo (citado en Mansilla, 2007, p. 48), Tafur (1994, pp. 1314), Agreda (2007, pp. 106-108) y Álvarez de Zayas (2012, p. 165), cada uno de estos autores, desde su punto de vista y formación filosófica y científica respectiva, manifiestan el proceso histórico del concepto de epistemología. Algunos de ellos, cabe mencionar, hacen un tratamiento teórico comparativo entre lo que es la epistemología y la teoría del conocimiento, si bien ambas se vinculan a procesos de generación de saberes y conocimientos a través de diversos métodos científicos, de acuerdo con doctrinas específicas y universales.

\section{Discusión y análisis sociocrítico}

Para el presente trabajo es necesario determinar el contexto teórico conceptual que debe tener la palabra epistemología. En esa línea, primeramente se requiere hacer un tratamiento analítico de la fuente de origen de la palabra, y eso se realiza a través de la etimología. Según Fernández (citado en Sánchez, 1996, p. 553), dicha palabra proviene del griego epistéme, que significa 'conocimiento, saber científico, y logos tratado'. Los diccionarios de Encarta (2005) y Larousse (1978, p. 413) mencionan que epistemología (del griego غ̇ logía) es doctrina de los fundamentos y métodos del conocimiento científico.

De acuerdo con estas referencias teóricas, episteme significa 'saber', y saber es sinónimo de "conocer (conocimiento), dominar y entender" (Karten, 1985, p. 323). Y la palabra logía significa 'tratado, estudio'; entonces, la epistemología, de acuerdo con su etimología, es el tratado o realización de estudio respecto al saber o al conocer; la acción de realizar el estudio es escudriñar y explicar las diversas formas de saber o conocer, de tal forma que se está generando 


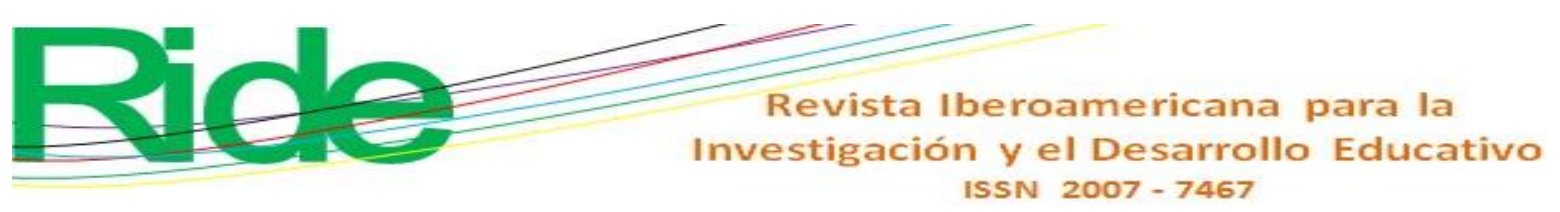

teoría respecto al saber o conocer, que en última instancia es la misma teoría del conocimiento. En ese sentido, por ejemplo, el contenido del diccionario de Sánchez (1996, p. 1339) entiende como sinónimos a epistemología y teoría del conocimiento.

La epistemología tiende a respetar espacios etimológicos con la finalidad de no confundir lo que es el saber y lo que es el conocimiento. Esta última se debe a la etimología griega gnosis ('conocimiento') y episteme, que es 'saber'. De esa forma, se aleja justificadamente de conocimiento. Entonces, epistemología es el estudio del saber, pero, a su vez, la epistemología es una rama del saber filosófico mayor (Eid, 2005). Con esto la epistemología se aleja aún más del conocimiento, porque el conocimiento es gnoseología como parte de la otra rama de la filosofía mayor; pero es más, porque, de acuerdo con el filósofo Ferrater (1964, p. 600), la palabra saber está relacionada con sabor y eso indica que se trata de "probar" las cosas y ver lo que "saben". Esta referencia da a entender que episteme o la palabra saber está más en contacto con la realidad; el concepto conocimiento no tanto así. Por ese motivo, se llega a entender provisionalmente que la epistemología es el estudio más del saber que del conocimiento mismo.

Aunado a lo anterior, la epistemología tiene una connotación más, con lo cual cumple una doble función en la teoría filosófica. Primero — como ya se dijo - significa 'el estudio del saber'; y segundo, es actuar como método. Esto por el siguiente motivo: el solo hecho de que la epistemología esté en contacto con la realidad, como fue descrita anteriormente, hace que sea el medio por el cual llegar al saber o generar conocimiento, ese medio, en otras palabras, es procedimiento, camino y también, en definitiva, es método. Las justificaciones vienen por los conceptos que tienen varios autores citados tanto en lo que refiere a gnoseología como a epistemología y por una cuestión de naturaleza teórica. En ese sentido, primero se evidenció que las definiciones de epistemología de la mayor parte de los autores citados coinciden en tomar en cuenta las palabras saber, conocimiento y metodología, ahí está la primera justificación; segundo, en ninguna parte de la estructura de la filosofía se podrá encontrar una rama que represente y responda a las siguientes interrogantes: cómo y con qué generar saberes o conocimientos y quién estudia los métodos de la filosofía, de las ciencias y los mismos métodos de la epistemología. Así, se entiende que los escritores sobre filosofía, los mismos filósofos, obviaron incluir la esencia del método e investigación para llegar al saber o conocimiento (eso es un error filosófico fenoménico y universal), por tanto, hasta la misma gnoseología necesita método. Ahora, si la filosofía tiene métodos (mayéutica y otros), estos son de carácter filosóficos, que poco o nada sirve a las múltiples y diversas filosofías y ciencias de la presente época. Tampoco lo que 


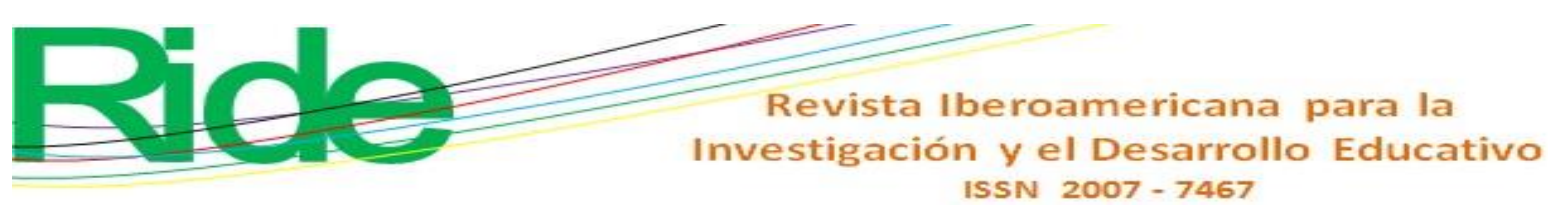

Aristóteles (siglo III a. C.) consideraba significar en cuanto a que episteme es "la forma de saber y alcanzar la verdad" (Conill, citado Sánchez, 1996, p. 547) de alguna forma cubre la falencia detectada porque da a entender la preocupación solo de "alcanzar la verdad", pero la verdad es solo una parte de los saberes y conocimientos. Entonces, en suma, la epistemología estudia el saber y los métodos.

\section{Proposición de definición del concepto de epistemología}

La epistemología es la rama de la filosofía que tiene el propósito de estudiar el saber, el conocimiento, los métodos y la respectiva validez, con los cuales se generaron y generan nuevos saberes y conocimientos tanto para la misma filosofía como para las diversas ciencias.

\section{Del concepto de teoría del conocimiento}

Las diferentes referencias bibliográficas indican que la teoría del conocimiento es la misma epistemología, y sugieren dirigirse a consultar las teorías de la epistemología, el conocimiento y la gnoseología. Con esta referencia, inicialmente se podría admitir que la teoría del conocimiento es igual a epistemología y gnoseología. Vale la pena, sin embargo, ir directamente a las fuentes.

El filósofo y sacerdote Hessen (1999) en su texto primera versión original escrito en 1925 cuyo título es Teoría del Conocimiento, utiliza indistintamente las palabras teoría del conocimiento y epistemología, aunque aclara que "la teoría del conocimiento es, como su nombre indica, una teoría, esto es, una explicación e interpretación filosófica del conocimiento humano" (p. 23).

Por su parte, los autores rusos Rosental y Iudin (2007), en un acápite dedicado a la epistemología y teoría del conocimiento, señalan a estas palabras en el sentido de "teoría del conocimiento, gnoseología. El término de 'epistemología' se emplea en la filosofía burguesa británica y norteamericana; es menos frecuente en la francesa y alemana" (p. 148).

Para García (citado en Sánchez, 1996) la teoría del conocimiento es lo siguiente:

El estudio del conocimiento desde el punto de vista filosófico es abordado por la disciplina denominada como gnoseología o teoría del conocimiento que intenta dar respuestas y soluciones a distintos problemas: planteamiento y validez del conocimiento, criterio de certeza, límites, formas del conocimiento, grados de seguridad y expresión de los mismos mediante el lenguaje (p. 308) 


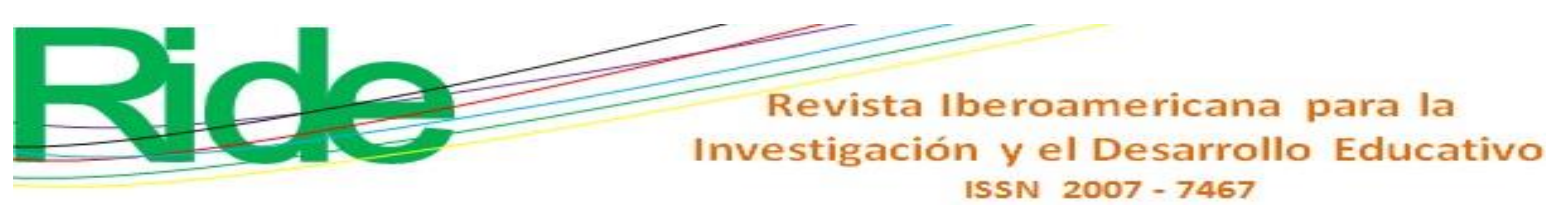

Discusión y análisis sociocrítico

Hessen (1999) utiliza el nombre de epistemologías para referirse a su vez a términos como dogmatismo, escepticismo, subjetivismo, relativismo, pragmatismo y criticismo, los cuales corresponden a la posibilidad del conocimiento. También este autor, en lugar de recurrir a epistemología, utiliza el nombre de teoría del conocimiento. Sin embargo, esto tiene su explicación y justificación, porque según Rosental y Iudin (2007), ya que los alemanes (como Hessen) utilizan el nombre de teoría del conocimiento y no la palabra epistemología para referirse a los seis términos señalados. En ese sentido, las referencias teóricas proporcionan una idea aproximada de lo que es la teoría del conocimiento. Primero se comparte el concepto que da Hessen; segundo, que cualquier grupo de filósofos, científicos u otros son libres de utilizar los términos de teoría del conocimiento, epistemología o gnoseología, y tercero, es recomendable entender que la teoría del conocimiento es más gnoseología que epistemología.

\section{Proposición de definición del concepto de teoría del conocimiento}

La teoría del conocimiento es la misma gnoseología de la filosofía, cuyas teorías se utilizan de acuerdo con el interés del grupo de filósofos, científicos u otros; es sinónimo -pero alejado- de la epistemología.

\section{Discusión general y propuesta de reconceptualización de gnoseología, epistemología y teoría del conocimiento}

De acuerdo con el estudio de cada uno de los términos filosóficos de gnoseología, epistemología y teoría del conocimiento, se llega a percibir los siguientes aspectos:

- Existencia de diferencias de fondo entre la gnoseología y la epistemología.

- $\quad$ Existencia de similitudes entre la teoría del conocimiento y la gnoseología; llegan a ser sinónimos por esencia.

- Que la epistemología cumple doble función: estudio del saber/conocimiento y estudio del método.

- La epistemología es método para todas las ciencias y filosofías.

- Existen diferencias superficiales entre los tres términos filosóficos, lo cual indica que son sinónimos.

- $\quad$ Cada uno es un concepto diferenciado. 


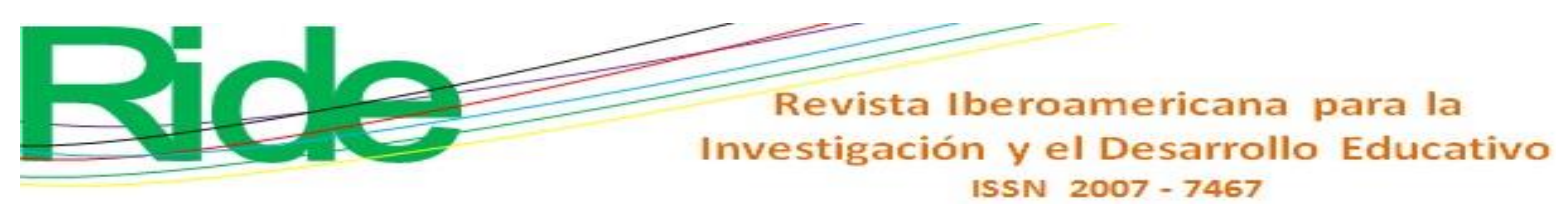

El tema de la comprensión y explicación, primeramente ubica este problema conceptual en la historia de la época Helénica y Galileana donde existía el positivismo y antipositivismo, y en el siglo XIX en el dilema y dicotómico de Ciencias Naturales y Ciencias del Espíritu, donde las primeras utilizarían el método de explicación y los segundos el método de la comprensión. (pp. 615-616)

Finalmente, Martínez (2006) comparte la idea aquí arriba inmediatamente mencionada, aunque es más explícito y claro en su posición:

El espíritu de toda esta orientación epistemológica no es nuevo, pues nos viene desde finales del siglo XIX, cuando Dilthey, Spranger, Weber, Jaspers y otros teóricos germánicos distinguieron claramente entre explicar (erklären) y comprender (verstehen). Los conceptos son los siguientes:

La explicación se centra en el análisis y en la división para buscar las causas de los fenómenos y su relación y semejanza con otras realidades, con las cuales es comparada, referida y relacionada, es decir, "insertada en leyes más amplias y universales", y que tiene más aplicación en las ciencias de la naturaleza. Las relaciones que establece pueden permanecer, sin embargo, exteriores a los objetos analizados; no conducen a su naturaleza.

La comprensión, por el contrario, es la captación de las relaciones internas y profundas mediante la penetración en su intimidad, para ser entendida desde adentro, en su novedad, respetando la originalidad y la indivisibilidad de los fenómenos. En lugar de parcelar lo real, como hace la explicación, la comprensión respeta su totalidad vivida; así el acto de comprensión reúne las diferentes partes en todo compresivo y se nos impone con clara evidencia (p. 79).

\section{Discusión y análisis sociocrítico}

Schleiermacher (citado en Martínez, 2006) y Dilthey (citado en Martínez, 2006) se refieren exclusivamente al término comprensión, e indican que para llegar a comprender es necesario moverse en un círculo hermenéutico (que luego, como hemos dicho, pasa a ser un círculo virtuoso), en un constante retorno y vaivén del todo a las partes y de estas al todo, es tratar de comprender al autor mejor de lo que él mismo se habría comprendido. Morín (1999) indica que la comprensión es útil en ciencias sociales y humanas, en tanto que la explicación sirve para ciencias naturales y exactas, como también para la intelectualidad y su relación con el objeto. De 


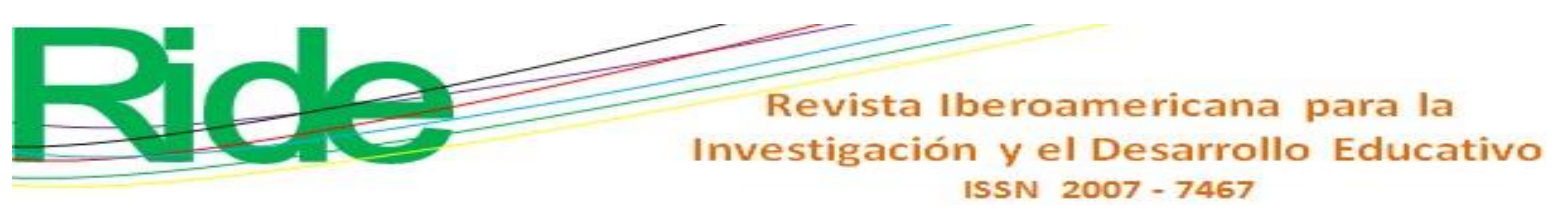

manera similar lo es para Vázquez (citado en Sánchez, 1996). Por último, para Martínez (2006) en el acto de la explicación es necesario dividir o parcelar la realidad, mientras que para la comprensión se debe tomar en cuenta la totalidad de los hechos sin dividirla.

Ahora, entrando a la discusión y análisis sociocrítico de los vocablos comprensión y explicación, con actitud psicopedagógica crítica y enfoque epistémico, el cual es el propósito de este trabajo, se hace necesario inicialmente plantear interrogantes que contribuirán a conocer algunos pormenores epistémicos que tienen ambos conceptos. En ese sentido, se plantean las siguientes interrogantes, que posteriormente serán discutidas y tratadas con el afán de dar respuesta a cada uno de ellas. Las interrogantes son:

1) ¿Por qué hacer tratamiento de los temas de explicación y comprensión?

2) ¿El propósito de la explicación y comprensión es llegar al entendimiento?

3) Entonces, ¿cuál es la esencia de la explicación y comprensión?

4) ¿La comprensión y explicación son una dicotomía inseparable?

5) ¿Por qué la explicación no es pertinente para ciencias sociales y la comprensión no es pertinente para ciencias exactas o naturales?

6) ¿La explicación y la comprensión abarcan la pluridiversidad de los hechos?

7) ¿Cuál es la propuesta conceptual que aglutina a los términos explicación, comprensión y entendimiento?

En estas siete interrogantes centraremos nuestra discusión sociocrítica del tema.

De la primera interrogante, “¿Por qué hacer tratamiento de los temas de explicación y comprensión?", cabe mencionar que la vida del ser humano está rodeada de objetos materiales y de fenómenos que la gobiernan, así como el alma, el espíritu, las acciones de las costumbres, tradiciones, los valores, las creencias, las tendencias religiosas y otros similares. Para la búsqueda de la felicidad, necesariamente el ser humano debe saber y conocer los componentes objetivos y subjetivos que presenta cada uno de los elementos que le rodean, y entre ellos se encuentra también el prójimo, por tanto, para saber, conocer y luego entender es necesario recurrir al acto de la explicación y comprensión de los hechos. Sin la acción de hacer la explicación y la comprensión no sería tan fácil entender dicha realidad de nuestro entorno. Por ese motivo, la explicación y la comprensión de los hechos son bastante relevantes para el desenvolvimiento de la vida del ser humano. 


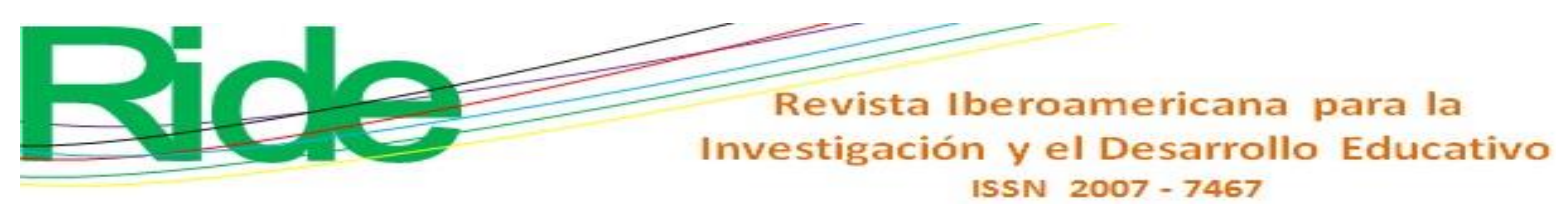

Respecto a la segunda interrogante, “¿El propósito de la explicación y comprensión es llegar al entendimiento?", el entendimiento implica haber captado, haber retenido, haber digerido mentalmente, haber conocido y saboreado el hecho en cuestión, y para llegar a ese estado de cognición es necesario pasar por recibir la explicación y/o la comprensión, por tanto, estos dos actos tienen el propósito de dotar al ser humano de los elementos cognitivos y epistemes para el dominio del entendimiento de los hechos. Así, el propósito de la explicación y comprensión es contribuir al entendimiento de los hechos.

De la tercera interrogante, "Entonces, ¿cuál es la esencia de la explicación y comprensión?", el contenido de esta no cuestiona el simple hecho de hacer el tratamiento conceptual de ambos términos, sino exige escudriñar sus componentes hasta alcanzar a conocer su esencia o el núcleo que le da vida, o mostrar el ser de lo que es. En consecuencia, hay que aclarar que explicación es sinónimo de explanación, esclarecimiento y es demostración del tema en cuestión, para ello se recurre a dividir, separar, descuartizar y hacer similares a los hechos de la vida material o no material, y en ese proceso se van conociendo los lazos de unión que tiene cada uno de los componentes con los otros. Asimismo, se van conociendo las funciones que cumplen al interior del todo estructurado, y para llegar a entenderlo, lo separado se vuelve a unir, a formar parte de un entramado, para que una vez culminado y conformado nuevamente se diga “¡ahora sí entiendo!”. Este procedimiento de la explicación es propicio para objetos materiales, como se señalaba anteriormente, pero también es propicio para temas sociales y culturales, como casos de espiritualismo, valores, creencias, prácticas costumbristas, acciones mentales y otros similares. Aquí, sin embargo, se debe de hacer notar lo siguiente. En temas socioculturales, el proceso de la explicación viene entrelazado o mezclado con el componente humano subjetivo, cuya significación es más relevante que la simple explicación material, de esa forma también y paulatinamente se va pasando a la comprensión, término que, en criterio de algunos de los autores citados, está separado de la explicación. En ese sentido, la esencia de la comprensión es abrazar todos los elementos del hecho, es escudriñar su composición buscando a la vez los elementos significativos y trascendentales subjetivos que hacen que el hecho exista y se manifieste en un entorno determinado. En síntesis, es ver a los hechos en sus componentes íntimos profundos. Por otro lado, la esencia de la explicación es ver a los hechos en sus componentes más objetivos y superficiales. 


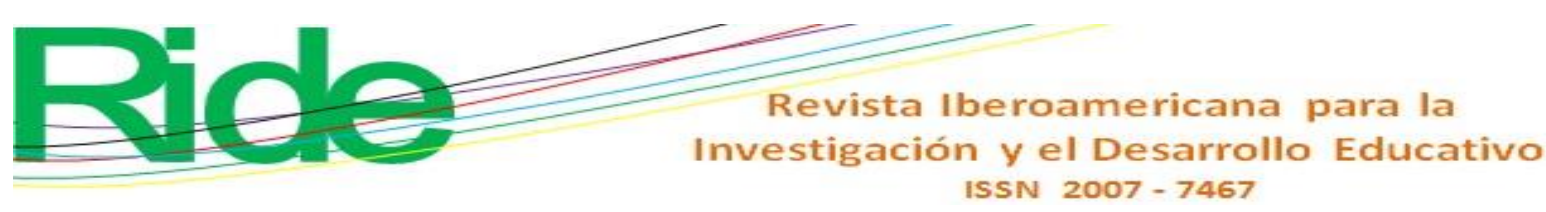

En cuanto a la cuarta interrogante, “¿La comprensión y explicación son una dicotomía inseparable?", la respuesta directa es no, puesto que pueden servir de modo separado y cada uno puede contribuir según su estilo al entendimiento particularizado, pero para un entendimiento completo es necesario no separarlos. Así, por ejemplo, la explicación puede contribuir relevantemente en ciencias como las naturales, físicas y exactas, sin la presencia de la comprensión, y así llegar al entendimiento, puesto que en estas prevalece la materialidad, la deducción simple de las hechos y acontecimientos, donde las cantidades, costos, tiempo y espacio son las causas para basarse solo con una simple explicación. Por tanto, en dichas ciencias, la comprensión que es signo de subjetividad, inducción y humano no tiene relevancia significativa; la autonomía de la explicación es como discriminación noble por la naturaleza de dichas ciencias. En que tanto la comprensión no discrimina. La comprensión, por naturaleza, puede actuar de modo separado como también puede actuar de forma conjunta y holísticamente con la explicación. Actúa profundamente cuando trata temas exclusivamente de ciencias sociales y culturales, pero cuando trata temas de ciencias de la naturaleza y similares necesariamente recurre primero a la explicación.

Ahora, respondiendo a la interrogante, para casos de estudio orientados al entendimiento de los hechos, la explicación y la comprensión pueden separarse y cada una responder relevantemente a sus respectivas ciencias: aquel puede prescindir de la comprensión para llegar al entendimiento, y esta, la compresión, puede proceder de la misma forma, lo cual significa que ambas no forman una dicotomía inseparable.

De la quinta interrogante, “¿Por qué la explicación no es pertinente para ciencias sociales y la comprensión no es pertinente para ciencias exactas o naturales?”, desde sus orígenes epistémicos, la explicación fue utilizada semánticamente en ciencias exactas, físicas y naturales (materialismo cuantitativo), en tanto que la comprensión denota hacer el esfuerzo humano no solo de entender los hechos socioculturales, sino también comprender las intersubjetividades que existen en las relaciones sociales y las relaciones materiales trascendentales.

La comprensión no es pertinente para ciencias exactas o naturales porque para estas ciencias no son relevantes los elementos subjetivos y trascendentales que tienen los objetos materiales. Ahora, la explicación para las ciencias sociales no es suficiente como para llegar a comprender y entender las cuestiones subjetivas y trascendentales que tiene la naturaleza y la vida humana. 


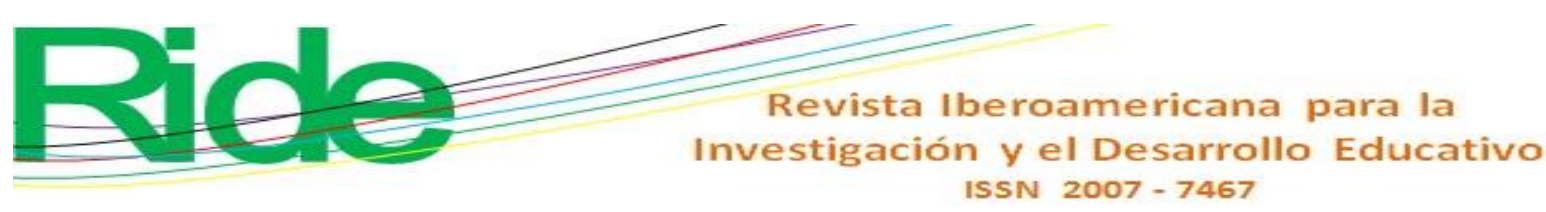

De la sexta interrogante, “¿La explicación y la comprensión abarcan la pluridiversidad de los hechos?", se pudiera responder que sí, pero de forma separada. Mayor relevancia asume la comprensión porque le interesa, desde un enfoque holístico, la subjetividad y trascendencia de la materia objetiva, pero particularmente de los hechos sociales y culturales. La explicación, por su parte, generalmente centra su atención en objetos materiales y poco o nada le interesa la subjetividad de la materia y de los hechos sociales. En cuanto a la pluridiversidad, ambos conceptos abarcan el tratamiento de la pluralidad de hechos materiales y humanos, asumiendo todos los campos de la diversidad, pero la comprensión es la que abarca más la pluridiversidad porque dicho término es sinónimo de totalidad, de holístico: integralidad de todos los componentes de los hechos.

De la séptima interrogante, “¿Cuál la propuesta de concepto que aglutina a los términos de explicación y comprensión?", como se habrá advertido en la exposición de las anteriores respuestas, tanto una como otra se diferencian, pero en algunos casos funcionan dicotómicamente, por ese motivo es posible plantear un concepto que aglutine a ambos términos.

\section{Proposición conceptual aglutinadora}

La comprensión es el modo cualitativo de entender, asimilar, aprehender cognitivamente los significados de los hechos objetivos, subjetivos y trascendentales y escritos epistémicos a través del tratamiento hermenéutico, respetando las relaciones internas, profundas, de la originalidad y la indivisibilidad de los actores de fenómenos teóricoepistemológicos, con apoyo de la historia y el procedimiento de la explicación. 


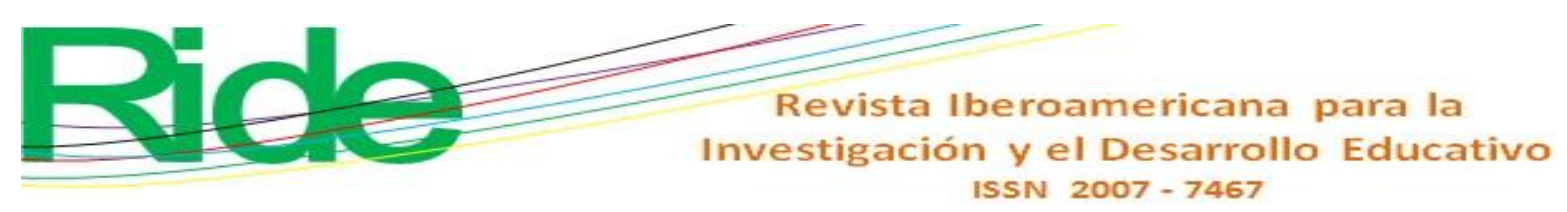

\section{Conclusión}

El objetivo del presente artículo fue proponer nuevos conceptos de términos epistémicos que son utilizados en el ámbito universitario y particularmente en ciencias socioculturales. Esto se cumplió al asumir el tratamiento de los términos gnoseología, epistemología, teoría del conocimiento, explicación y comprensión. Con ese propósito inicialmente fue elaborado el concepto de psicopedagogía crítica, que contribuyó pertinentemente para el tratamiento de los conceptos epistémicos y sociales, y con el cual se culminó satisfactoriamente con la proposición de nuevos conceptos. Asimismo, se detectó que cada uno de los autores aquí citados tiene su posición conceptual respecto a un solo término universal, lo cual lleva a confusión.

Por otro lado, se detectó que las tres epistemes de la gnoseología, epistemología y teoría del conocimiento son sinónimas, pero la epistemología se diferencia de las otras dos por ser la única que hace tratamiento del tema del método. Y respecto a los términos sociales de explicación y comprensión, ambos pueden contribuir de forma separada y de forma conjunta a las ciencias físicas, naturales y sociales, pero este de alguna forma se diferencia de aquel porque es más profundo su actuar en relación con todo tipo de hechos, incluso llega a ver cuestiones objetivas, aunque principalmente se enfoca en temas intersubjetivos y trascendentales de todos los hechos materiales y humanos.

Finalmente, esta investigación dejó dos interrogantes que pueden ser expresados de la siguiente forma: ¿deben existir los conceptos específicos de gnoseología y epistemología para cada una de las diversas ciencias? y ¿el concepto de explicación propuesto será adecuado para las ciencias espirituales? En ese sentido, se invita a los interesados en esta temática abordada a asumir el reto de responder a través de la investigación a ambas interrogantes. 
